\title{
Biophysical Synaptic Dynamics in an Analog VLSI Network of Hodgkin-Huxley Neurons
}

\author{
Theodore $\mathrm{Yu}^{1,4}$ and Gert Cauwenberghs $\mathrm{s}^{2,3,4}$ \\ ${ }^{1}$ Electrical and Computer Engineering Department, Jacobs School of Engineering \\ 2 Neurobiology Section, Division of Biological Sciences \\ ${ }^{3}$ Bioengineering Department, Jacobs School of Engineering \\ ${ }^{4}$ Institute for Neural Computation \\ University of California San Diego, La Jolla, CA 92093
}

\begin{abstract}
We study synaptic dynamics in a biophysical network of four coupled spiking neurons implemented in an analog VLSI silicon microchip. The four neurons implement a generalized Hodgkin-Huxley model with individually configurable rate-based kinetics of opening and closing of $\mathrm{Na}+$ and $\mathrm{K}+$ ion channels. The twelve synapses implement a rate-based firstorder kinetic model of neurotransmitter and receptor dynamics, accounting for NMDA and non-NMDA type chemical synapses. The implemented models on the chip are fully configurable by 384 parameters accounting for conductances, reversal potentials, and pre/post-synaptic voltage-dependence of the channel kinetics. We describe the models and present experimental results from the chip characterizing single neuron dynamics, single synapse dynamics, and multi-neuron network dynamics showing phaselocking behavior as a function of synaptic coupling strength. The $3 \mathrm{~mm} \times 3 \mathrm{~mm}$ microchip consumes $1.29 \mathrm{~mW}$ power making it promising for applications including neuromorphic modeling and neural prostheses.
\end{abstract}

\section{INTRODUCTION}

The biological system observed in animal behavior is incredible in its range and depth of function. This is especially apparent in the capacity of the brain and neuromuscular system to govern and control the body in its vast assortment of tasks. Neuromorphic engineering [1] takes inspiration from neurobiology in the design of artificial neural systems in silicon integrated circuits, based upon function and structural organization of biological nervous systems. By emulating the form and architecture in biological systems, neuromorphic engineering seeks to emulate the function as well [3], [4], [8], [9] and [10]. In the context of the neuromuscular system, this leads to the study of neural networks and synaptic dynamics. Not only does this prove helpful to advance the knowledge and understanding of the biological models, but they can also be used to interface with biological systems as a prosthesis.

Here we present a network of Hodgkin-Huxley [2] neurons and conductance-based synapses that accurately models the detailed rate-based kinetics of membrane channels in the neural and synaptic dynamics. The voltage dependence of the channel opening and closing rates is approximated through programmable analog regression functions. Circuit details of the NeuroDyn system are presented in a previous work [13]. The synaptic dynamics and coupled neurons which were not
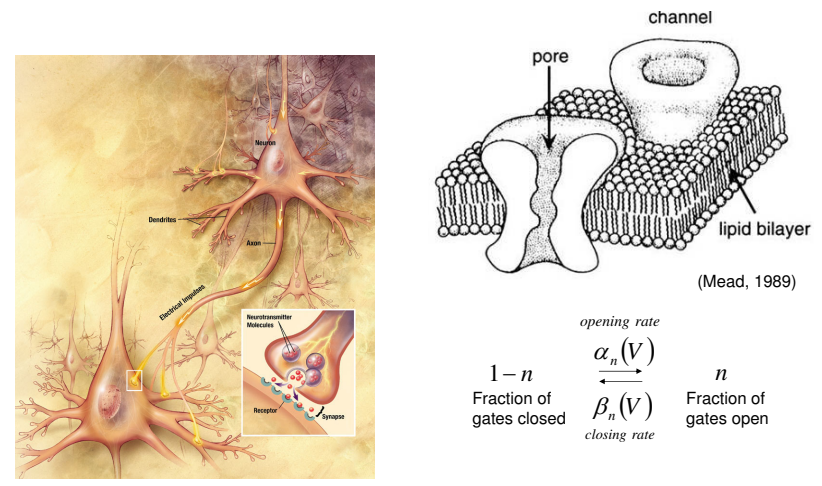

Fig. 1. A neuron is pictured with inset detail showing synaptic neurotransmitter transfer (left, source is Wikipedia.com). A membrane channel is pictured (source is Mead, 1989) with an accompanying mathematical expression describing the channel kinetics.

presented in [13] are described later in this paper. The biorealistic accuracy derived from a biophysical origin basis for the model implementation of the neurons and synapses coupled with the flexibility offered with a programmable interface makes the NeuroDyn system well-suited to model and simulate a variety of biological systems. For example, central pattern generators require only a few neurons for implementation yet they can characterize complex behavior [7]. Furthermore, there are various analog and digital exposed probes in the circuit board allow for a real-time interface to the internal membrane channel dynamics. Previous neuron-based circuit systems have been used to either model or interface to various biological systems through the implementation of sophisticated signal processing [5], [6]. The NeuroDyn system provides greater insight into the biological basis behind the function through the biophysical origin of its models.

\section{A. NeuroDyn Overview}

The NeuroDyn Board consists of 4 Hodgkin-Huxley based neurons fully connected through 12 conductance-based synapses as shown in Fig. 2(b). All parameters are individually addressable and individually programmable and are biophysically-based governing the conductances, reversal po- 

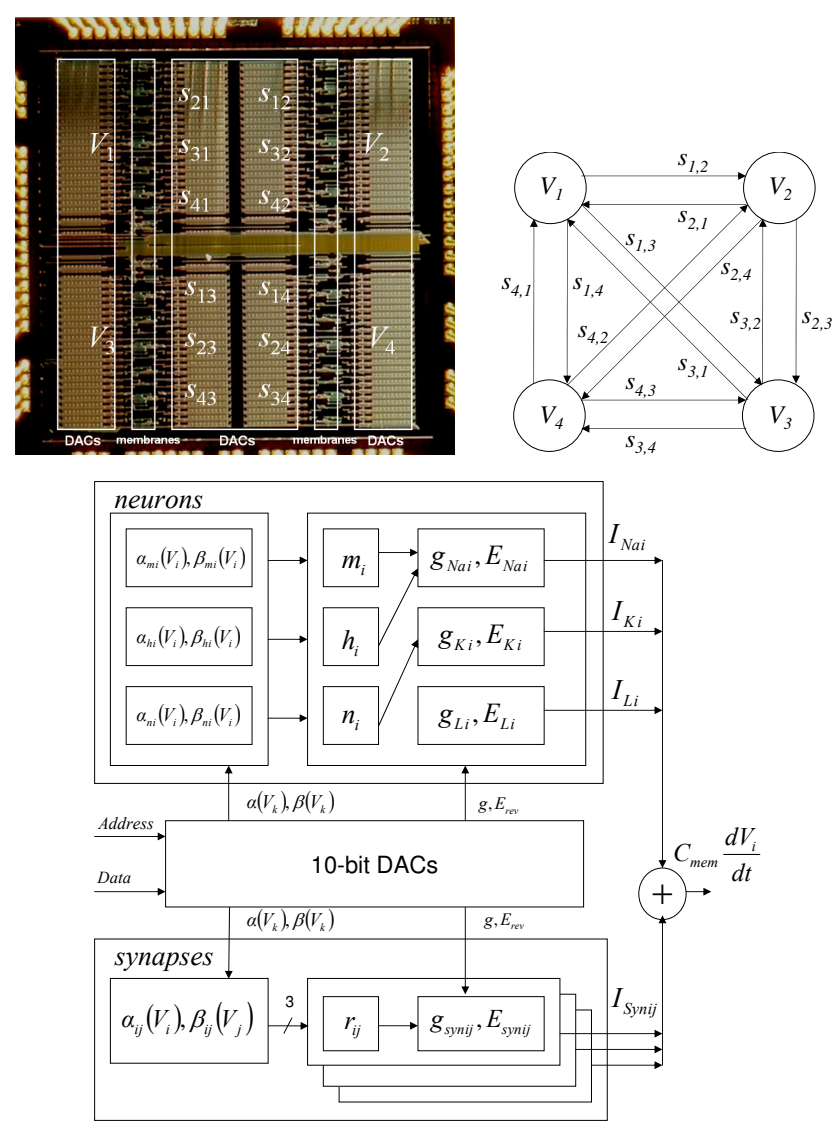

Fig. 2. NeuroDyn chip micrograph (top left) and system diagram (top right). Four neurons are interconnected by twelve synapses, each with programmable channel kinetics, conductances, and reversal potentials (see Table 1). System diagram for one of the four neurons in the NeuroDyn chip (bottom).

TABLE I

NeURoDyn DAC PARAMETERS

$\begin{array}{rccc}\text { Neurons } \mathrm{V}_{i}: & & & \\ \alpha_{n_{i}}(V) & \beta_{n_{i}}(V) & \mathrm{g}_{N a_{i}} & \mathrm{E}_{N a_{i}} \\ \alpha_{m_{i}}(V) & \beta_{m_{i}}(V) & \mathrm{g}_{K_{i}} & \mathrm{E}_{K_{i}} \\ \alpha_{h_{i}}(V) & \beta_{h_{i}}(V) & \mathrm{g}_{L_{i}} & \mathrm{E}_{L_{i}} \\ 4 \times 3 \times 7^{*} & 4 \times 3 \times 7^{*} & 4 \times 3 & 4 \times 3 \\ \text { Synapses } \mathrm{s}_{i j}: & & & \\ \alpha_{r_{i j}}\left(V_{p r e}\right) & \beta_{r_{i j}}\left(V_{\text {post }}\right) & \mathrm{g}_{s y n_{i j}} & \mathrm{E}_{s y n_{i j}} \\ 12 \times 7^{*} & 12 \times 7^{*} & 12 & 12\end{array}$

*All rates $\alpha, \beta$ are functions of voltage as 7-point sigmoidal spline.

tentials, and voltage-dependance of the channel kinetics. There are a total of 384 programmable parameters governing the dynamics as shown in Table I. Each parameter is stored onchip in a 10-bit DAC.

\section{B. Chip Architecture}

The NeuroDyn chip is organized into four quadrants with each quadrant containing one neuron, its associated membrane capacitance and the three synaptic inputs from the other neurons. Notice that each channel current and synaptic current follow the same general form as illustrated in Fig. 2(c). The channel current is a product of a conductance term modulated by a product of gating variables and the difference between the membrane voltage and reverse potential as illustrated below in (1) and (2). The similar form for both the neuron channel currents and synaptic current allow for a small number of circuits to model each component of the channel current.

\section{BIOPHYSICAL MODELS}

\section{A. Membrane Dynamics}

The Hodgkin-Huxley membrane dynamics including conductance-based synapses is described by

$$
C_{m e m} \frac{d V_{i}}{d t}=-I_{N a_{i}}-I_{K_{i}}-I_{L_{i}}-\sum_{j} I_{s y n_{i j}}
$$

where $i, j=0 \ldots 3$, and

$$
\begin{aligned}
I_{N a_{i}} & =g_{N a_{i}} m_{i}{ }^{3} h_{i}\left(V_{i}-E_{N a_{i}}\right) \\
I_{K_{i}} & =g_{K_{i}} n_{i}{ }^{4}\left(V_{i}-E_{K_{i}}\right) \\
I_{L_{i}} & =g_{L_{i}}\left(V_{i}-E_{L_{i}}\right) \\
I_{s y n_{i j}} & =g_{s y n_{i j}} r_{i j}\left(V_{i}-E_{s y n_{i j}}\right)
\end{aligned}
$$

\section{B. Channel Kinetics}

The gating variables $n_{i}, m_{i}$, and $h_{i}$ in the HH neuron model are modeled by a rate-based approximation to the kinetics governing the random opening and closing of membrane channels [11]:

$$
\frac{d n}{d t}=\alpha(1-n)-\beta n
$$

where $\alpha$ and $\beta$ are the voltage dependent opening and closing rates of the channel gates, and $n$ (which stands for any of the $n, m, h$, and $r$ variables) is the fraction of channel gates in the open state. In the case for the synapse dynamics, the rate variables $r_{i j}$ are modeled slightly differently:

$$
\frac{d r}{d t}=\alpha T(1-n)-\beta r
$$

where $\mathrm{T}=$ neurotransmitter concentration and is defined by:

$$
T=\frac{T_{\max }}{1+e^{-\left(V_{p r e}-V_{p}\right) / K_{p}}}
$$

where $r$ is the fraction of receptors in the open state, $T_{\max }$ is the maximum neurotransmitter concentration, $V_{\text {pre }}$ is the membrane potential of the presynaptic neuron, $V_{p}$ is the halfrise voltage potential, and $K_{p}$ controls the steepness of the sigmoid.

We are able to model both NMDA and non-NMDA type synapses using the programmable architecture described above. Each synaptic connections opening rate $\beta$ term is dependent upon the pre-synaptic membrane voltage. By setting the programmable values for the $\alpha$ and $\beta$ opening and closing functions, we can model NMDA and non-NMDA synapses. We can also vary the $E_{\text {syn }}$ value relative to the resting membrane potential to program each synapse as either inhibitory or excitatory. 

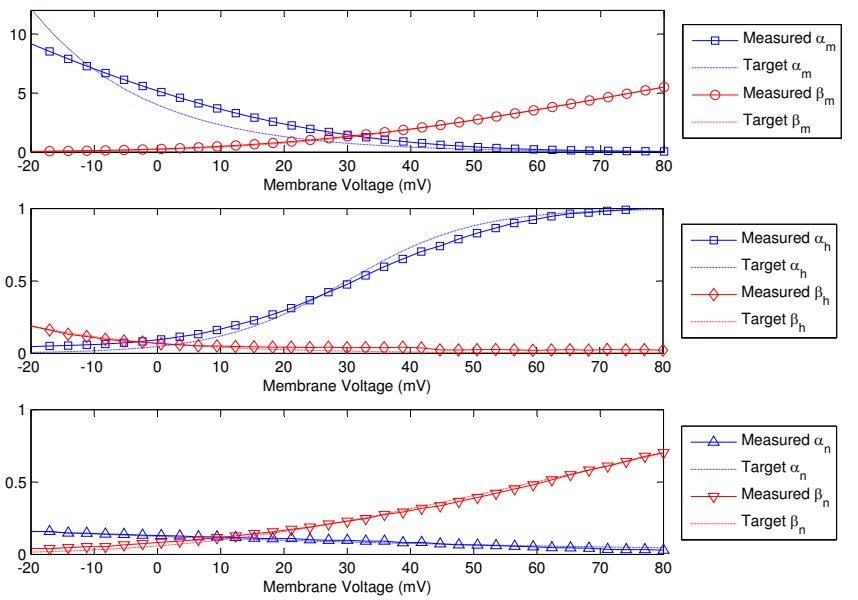

Fig. 3. Target and measured channel opening and closing rates $\alpha$ and $\beta$ for gating variables $n, m$, and $h$ of a $\mathrm{HH}$ neuron, obtained by fitting of the on-chip programmed parameters.

\section{Neuromorphic Modeling}

The membrane dynamics and general rate-based models of voltage-gated channel kinetics are emulated in silicon. We model the voltage dependence of each of the opening and closing rates as a 7-point sigmoidal spline regression function. The voltage bases of the splines are linearly spaced, and the amplitudes are individually programmable (including sign selection), allowing for large and varied model space.

\section{A. Programmable Channel Kinetics}

We programmed the gate opening and closing variables for one neuron implementing the $\mathrm{HH}$ model. We performed linear regression to determine the parameters of the spline model based on chip characteristics. With 10-bit programming for each of the 7 spline amplitude levels in the regression functions, an adequate fit was obtained as shown in Fig. 3.

\section{EXPERIMENTAL RESUlTS}

\section{A. Neuron Spiking Dynamics}

We implemented the HH model in one neuron and observed the dynamics of the membrane and gating variables as shown in Fig. 4.

\section{B. Synapse Dynamics}

We programmed a $G A B A_{A}$ inhibitory synapse with a single presynaptic spiking neuron input according to the configuration illustrated in Fig. 5(a). The conductance curves of the synapse are shown in Fig. 5(b). We chose to demonstrate synaptic dynamics using a simple network of two coupled neurons connected with inhibitory synapses as illustrated in Fig. 6. The neurons were programmed with parameters according to the equations of the Hodgkin-Huxley model. The synapses were programmed with parameters according to $G A B A_{A}$ inhibitory synapses.

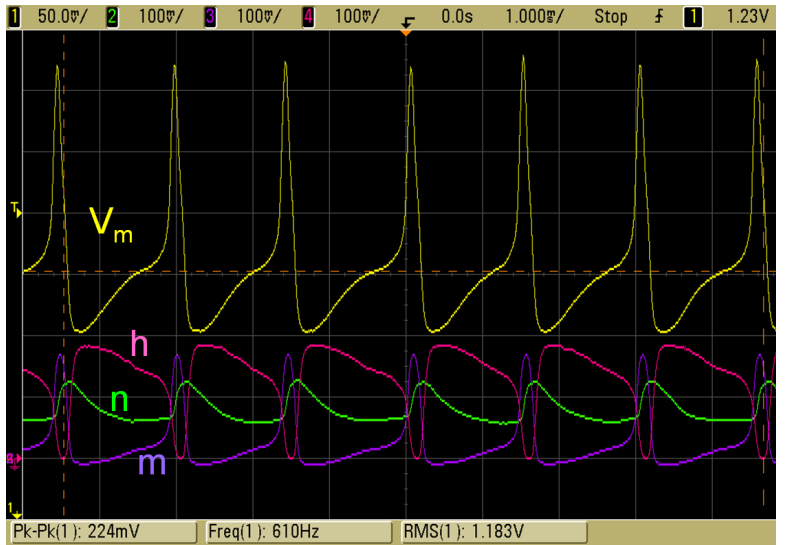

Fig. 4. Measured dynamics of membrane voltage $V_{m}$ and gating variables $n, m$, and $h$ for a single $\mathrm{HH}$ neuron.

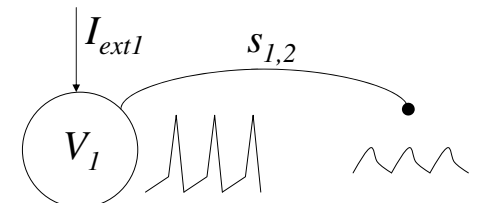

Spiking neuron synapse

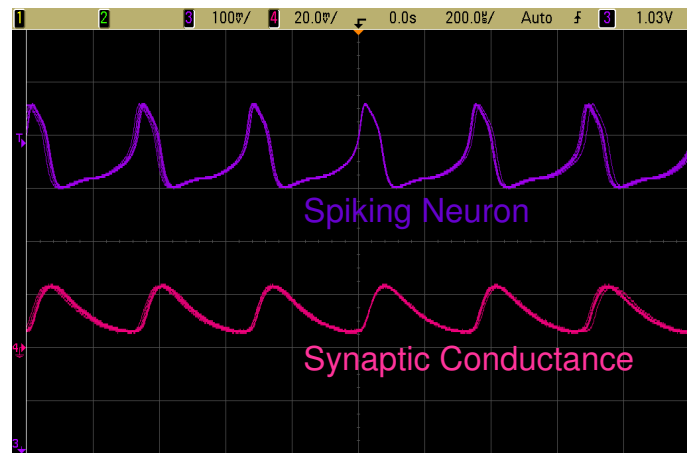

Fig. 5. (a) Synapse with presynaptic spiking neuron diagram (above). (b) Oscilloscope trace of the conductance curve of a synapse with a spiking presynaptic neuron input. Notice that the spiking neuron waveform (purple) and conductance curves for the synapse (pink) are in phase (below).

\section{Neuron Network Dynamics}

The neurons were first programmed to spike independently. All incoming synaptic inputs to each neuron were disconnected by setting the synaptic conductances to zero. Then the neurons were connected by setting the synaptic conductances to appropriate values. The resulting waveforms are shown in Fig. 7. Notice that especially in the oscilloscope capture from the couple neurons, that there is an observable timing jitter in the spiking neuron waveforms. This phase noise is due to the noise inherent in using a circuit implementation for the neuron and synapses. This proves to an advantage as it is more biorealistic to include random noise similar to the kind found in biological systems. 


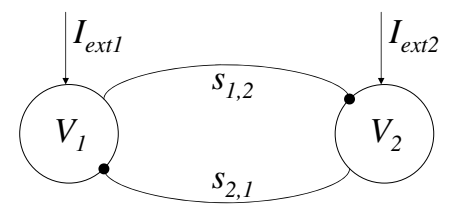

Fig. 6. Coupled neurons diagram. Two spiking neurons are connected with inhibitory synapses.

\section{CONCLUSion}

We present a fully connected biophysical network of neurons and synapses with digitally programmable analog membrane dynamics and channel kinetics. We demonstrated biorealistic results for neuron spiking dynamics and coupled neuron system with inhibitory synapses. All configurable parameters in the implemented model have a biophysical origin, thus supporting direct interpretation of the results of adapting/tuning the parameters in terms of neurobiology modeling. Future work involves the design and implementation of multi-compartment neurons.

\section{ACKNOWLEDGMENT}

The authors would like to thank Stephen Deiss and Mike Chi for help with the chip design and experimental setup. This work was funded by NIH R01AG029681. The design of the chip originated in a class project at UCSD, and the chip was fabricated through the MOSIS Educational Program. The authors would also like to acknowledge the 2008 NSF Workshop on Neuromorphic Cognition Engineering in Telluride, Colorado.

\section{REFERENCES}

[1] Mead, C.A., Analog VLSI and Neural Systems, Addison-Wesley. 1989.

[2] Hogdkin, A.L.; Huxley, A.F. "A Quantitative Description of Membrane Current and Its Application to Conduction and Excitation in Nerve," J. Physiol., vol. 117, pp. 500-544. 1952

[3] Andreou, A.; Boahen, K. "Translinear Circuits in Subthreshold MOS," Analog Integrated Circuits and Signal Processing, vol. 9, pp. 141-166. 1996

[4] Simoni, Mario F.; Cymbalyuk, Gennady S.; Sorensen, Michael E.; Calabrese, Ronald L.; DeWeerth, Stephen P. "A Multiconductance Silicon Neuron With Biologically Matched Dynamics," IEEE Transactions on Biomedical Engineering, vol. 51, no. 2. 2004

[5] Hudson, Tina A.; Bragg, Julian A.; Hasler, Paul; DeWeerth, Stephen P. "An Analog VLSI Model Of Muscle Contraction," IEEE Transactions on Circuits and Systems - II: Analog and Digital Signal Processing, vol. 50, no. 7. 2003.

[6] Hsiao, M.C.; Chan, C.H.; Srinivasan, V.; Ahuja, A.; Erinjippurath, G.; Zanos, T.P.; Gholmieh, G.; Song, D.; Wills, J.D.; LaCoss, J.; Courellis, S.; Tanguay, A.R. Jr.; Granacki, J.J.; Marmarelis, V.Z.; Berger, T.W. "VLSI Implementation Of A Nonlinear Neuronal Model: A 'Neural Prosthesis' To Restore Hippocampal Trisynaptic Dynamics," Proc. IEEE EMBS 2006, pp. 4396-4399. 2006.

[7] Tenore, F.; Etienne-Cummings, R.; Lewis, M.A. "A Programmable Array Of Silicon Neurons For The Control Of Legged Locomotion," Proc. IEEE ISCAS 2004, vol. 5, no. 349-352. 2004

[8] Farquhar, E.; Hasler, P. "A Bio-Physically Inspired Silicon Neuron," IEEE Transactions on Circuits and Systems - 1: Regular Papers, vol. 52, no. 3. 2005

[9] Georgiou, J.; Drakakis, E.M.; Toumazou, C.; Premanoj, P. ”An Analogue Micropower Log-Domain Silicon Circuit for the Hodgkin and Huxley Nerve Axon,” Proc. IEEE ISCAS 1999, vol.2, pp.286-289. 1999
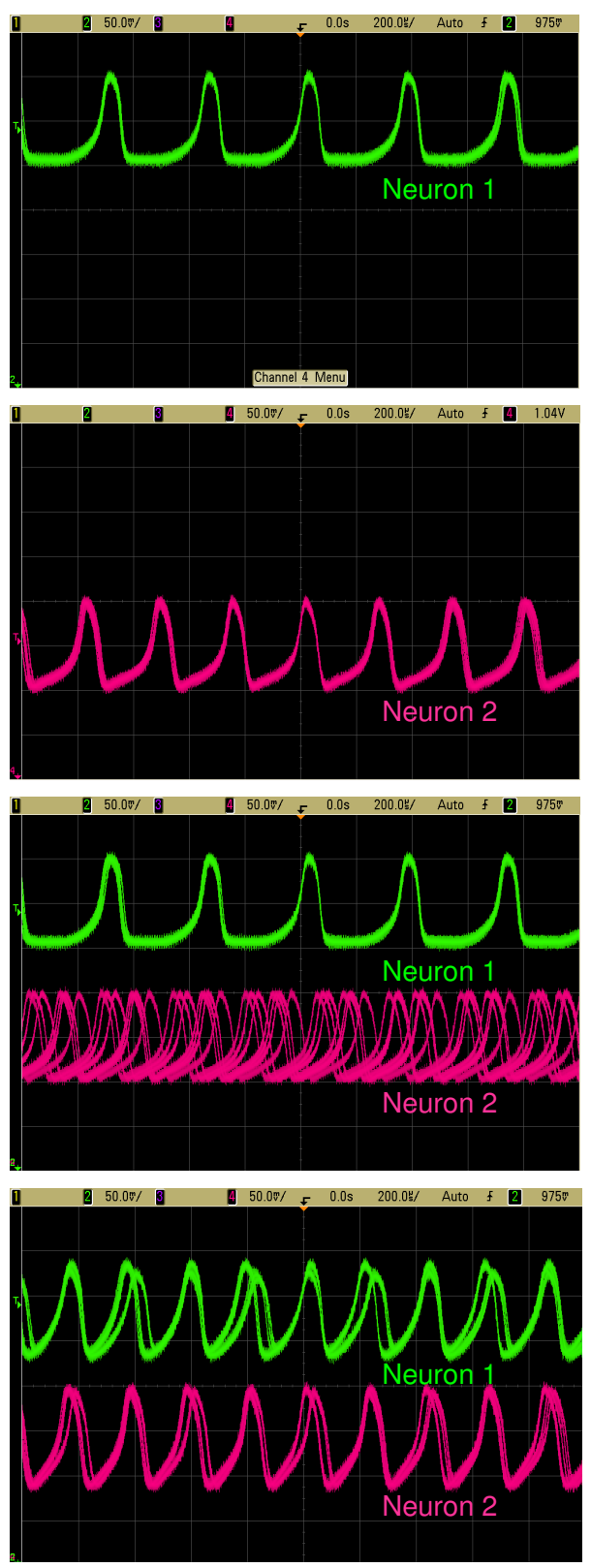

Fig. 7. Oscilloscope traces of coupled neurons. (a) and (b) show the individual spiking neurons. (c) shows the uncoupled neurons together. (d) shows the neurons coupled with inhibitory synapses spiking in synchrony.

[10] Saighi, S.; Bornat, Y.; Tomas, J.; Renaud, S. "Neuromimetric ICs and System for Parameters Extraction in Biological Neuron Models," Proc. IEEE ISCAS 2006, pp. 4207-4211. 2006

[11] Destexhe A., Z.F. Mainen and T.J. Sejnowski, "Synthesis of Models for Excitable Membranes, Synaptic Transmission and Neuromodulation Using a Common Kinetic Formalism," J. Comp. Neuroscience, vol. 1, pp. 195-230. 1994.

[12] Vogelstein, R.J.; Mallik, U.; Vogelstein, J.T.; and Cauwenberghs, G. "Dynamically Reconfigurable Silicon Array of Spiking Neurons With Conductance-Based Synapses," IEEE Trans. Neural Networks, vol. 18(1), pp. 253-265. 2007

[13] Yu, T.; Cauwenberghs, G. "Analog VLSI Neuromorphic Network with Programmable Membrane Channel Kinetics," Proc. IEEE ISCAS 2009, (in print) 